Finance Stochast. 1, 331-344 (1997)

Finance and
Stochastics
o Springer-verag 1997

\title{
Option pricing in the presence of natural boundaries and a quadratic diffusion term ${ }^{\star}$
}

\author{
Sven Rady \\ Graduate School of Business, Stanford University, Stanford, CA 94305-5015, USA \\ (e-mail: rady sven@gsb.stanford.edu)
}

\begin{abstract}
This paper uses a probabilistic change-of-numeraire technique to compute closed-form prices of European options to exchange one asset against another when the relative price of the underlying assets follows a diffusion process with natural boundaries and a quadratic diffusion coefficient. The paper shows in particular how to interpret the option price formula in terms of exercise probabilities which are calculated under the martingale measures associated with two specific numeraire portfolios. An application to the pricing of bond options and certain interest rate derivatives illustrates the main results.
\end{abstract}

Key words: Option pricing, bond options, change-of-numeraire technique, diffusion process, quadratic diffusion terms

JEL classification: G13

Mathematics subject classification (1991): 90A09, 60G35

\section{Introduction}

This paper studies the valuation of a European option to exchange one asset against another when the relative price of the underlying assets follows a diffusion process with natural upper and lower boundaries and possesses a diffusion

\footnotetext{
* This paper is based in part on a note entitled "Option Pricing with a Quadratic Diffusion Term: An Extension of Black-Scholes" which I presented at the London School of Economics (LSE) in 1993. A previous version of the paper was circulated as Discussion Paper No. 226, November 1995, of the LSE Financial Markets Group. I would like to thank Lucien Foldes, my Ph.D. advisor at the LSE, for his encouragement and guidance, Phil Dybvig and Steve Heston for stimulating discussions during my visit to Washington University, St. Louis, in December 1994, and Asbjørn Hansen, Ingrid Werner and three anonymous referees for their comments and suggestions. Financial support from the COLONIA Studienstiftung, the LSE Financial Markets Group and the Economic and Social Research Council of the UK is gratefully acknowledged.

Manuscript received: January 1996; final version received: December 1996
} 
coefficient which is a quadratic function of the current relative price. In contrast to the previous literature on variants of this problem, which calculates the option price by explicitly solving the fundamental valuation PDE, we use a purely probabilistic approach, based on a change-of-numeraire technique that goes back to Jamshidian (1987), El Karoui and Rochet (1989) and Geman (1989). This approach yields new insights on three levels. First, it provides a decomposition of the option price which holds whenever there are strict bounds on the relative price of the underlying assets. Second, it clarifies the structure of the pricing formulae obtained in the case of a quadratic diffusion term. Third, it unifies the pricing of bond options and certain interest rate derivatives for a particular class of underlying interest rate dynamics.

Our first result shows that with strict lower and upper bounds on the relative price of the underlying assets, the option price can be written as a weighted difference of the prices of two particular numeraire portfolios. The construction of these numeraires reflects the presence of the bounds, and the weight of each numeraire in the decomposition of the option price equals the probability, evaluated under the risk-neutral measure associated with the numeraire, that the option ends 'in the money' and is exercised.

Next, we prove the following characterisation result: modulo a simultaneous change of numeraire and martingale measure, assuming a quadratic diffusion term for the relative price of the underlying assets is equivalent to assuming that the relative price of the two numeraire portfolios follows a driftless geometric Brownian motion. Given this characterisation, it is straightforward to calculate the above exercise probabilities and hence the option price under the assumption of a quadratic diffusion term. ${ }^{1}$

Our specification of relative price dynamics with a quadratic diffusion term encompasses the exchange rate target zone model of Ingersoll (1989a, b) as well as the bond price model of Bühler and Käsler (1989). The bond price dynamics in lognormal models of the term structure of interest rates such as Sandmann, Sondermann and Miltersen (1994) also fit into this framework. ${ }^{2}$ Consequently, our formula for the price of an option to exchange one asset against another generalises the pricing formulae which the above authors derive for currency options and options on discount bonds, respectively. ${ }^{3}$

The application of the change-of-numeraire technique to options on discount bonds is particularly instructive, and we therefore present it in some detail. ${ }^{4}$ Now, the two assets under consideration are the bond on which the option is written, and a reference bond which expires at the same time as the option. The relative

\footnotetext{
${ }^{1}$ After completing the 1995 discussion paper I became aware of Beniamin Goldys' independent work on a purely probabilistic derivation of the option price, reported in Goldys (1995). His result will be presented in Section 4 below.

2 See also Brace et al. (1995) and Miltersen et al. (1997).

${ }^{3}$ See Sondermann $(1987,1988)$ for another approach to option pricing when there are bounds on the underlying security prices. Involving absorbing rather than natural boundaries, this approach leads to explicit valuation formulae of a different type.

${ }^{4}$ The application to Ingersoll's (1989a, b) target zone model with quadratic diffusion term can be found in the 1995 discussion paper.
} 
price of the underlying bond in units of the reference bond is simply the forward price of the underlying bond for delivery at the expiry of the option. We impose an upper bound equal to 1 on the forward bond price in order to guarantee positive forward interest rates. Applying the above characterisation result, we find that the forward bond price has a quadratic diffusion coefficient if and only if the corresponding once compounded forward rate follows a driftless geometric Brownian motion (under a suitably chosen martingale measure). Consequently, either of these conditions implies both Bühler and Käsler (1989) type pricing formulae for bond options and, by a standard calculation, Black (1976) type pricing formulae for simple caps and floors (i.e., call and put options) written on the forward rate. Thus, the probabilistic change-of-numeraire approach gives new insights into a link between valuation formulae which was first established by Sandmann, Sondermann and Miltersen (1994).

The paper is organised as follows. Section 1 sets out the framework of our analysis and presents the change-of-numeraire technique. Section 2 gives a general expression for the price of an option to exchange one asset against another when there are strict upper and lower bounds on the underlying relative price. Section 3 characterises models where the underlying relative price has a quadratic diffusion term, and then calculates the option price. Section 4 shows how the main results apply to the pricing of options on discount bonds and interest rate caps. Section 5 concludes the paper.

\section{Martingale measures and contingent claims}

Fix a finite time interval $\mathscr{T}=[0, T]$ and a filtered probability space $(\Omega, \mathscr{F}, P$, $\left.\left(\mathscr{T}_{t}\right)_{t \in \mathscr{T}}\right)$ satisfying $\mathscr{T}_{T}=\mathscr{F}$ and the usual conditions of completeness and right-continuity.

Consider a financial market with continuous and frictionless trade in two primitive assets, labelled 0 and 1 , which pay no dividends in $\mathscr{T}$. Let their price processes $S^{i}(i=0,1)$ be positive RCLL semimartingales on $\left(\Omega, \mathscr{F}, P,(\mathscr{T})_{t \in \mathscr{T}}\right)$. Relative security prices are given by the process $X=S^{1} / S^{0}$.

A probability measure $Q$ equivalent to $P$ is called a martingale measure with respect to asset 0 if $X$ is a $Q$-martingale. Alternatively, such a measure $Q$ is said to be risk-neutral with respect to asset 0 . Let $\mathbb{P}_{0}$ denote the set of these measures.

Assumption (M) $\mathbb{P}_{0}$ is non-empty.

Following Harrison and Pliska (1981), we shall hold one element of $\mathbb{P}_{0}$, denoted $Q_{0}$ and called the reference measure, fixed throughout the paper.

Given this reference measure, we can define the set $\Theta$ of admissible trading strategies as the set of all predictable vector processes $\theta=\left(\theta^{0}, \theta^{1}\right)$ such that $\theta^{1}$ is integrable with respect to $X$, the value process $V_{t}^{\theta}=\theta_{t}^{0} S_{t}^{0}+\theta_{t}^{1} S_{t}^{1}$ is non-negative, and the discounted value process $V^{\theta} / S^{0}$ is a $Q_{0}$-martingale satisfying 


$$
\frac{V_{t}^{\theta}}{S_{t}^{0}}=\frac{V_{0}^{\theta}}{S_{0}^{0}}+\int_{0}^{t} \theta_{s}^{1} d X_{s}
$$

(as usual, equalities between random variables are meant $P$-almost surely). As is well known, Assumption (M) and the construction of $\Theta$ ensure absence of arbitrage opportunities.

A positive process $N$ is called a numeraire if there is a trading strategy $\theta \in \Theta$ such that $N=V^{\theta}$. Extending our previous definition, we call a probability measure $Q$ equivalent to $P$ a martingale measure for numeraire $N$ (or riskneutral with respect to $N$ ) if $V^{\theta} / N$, the portfolio value expressed in units of the numeraire, is a $Q$-martingale for any strategy $\theta \in \Theta$. We shall write $\mathbb{P}_{N}$ for the set of all such measures, and $\mathbb{P}_{1}$ if $N=S^{1}$.

Given the measure $Q_{0}$ and a numeraire $N$, define a probability measure $Q_{N}$ equivalent to $Q_{0}$ (and hence to $P$ ) via the Radon-Nikodym derivative

$$
\frac{d Q_{N}}{d Q_{0}}=\frac{N_{T}}{N_{0}} S_{0}^{0} S_{T}^{0} .
$$

Note that $N / S^{0}$ is a $Q_{0}$-martingale by definition, so the right hand side of (1) has indeed expectation equal to one under $Q_{0}$. In case $N=S^{1}$, we shall write $Q_{1}$ for the measure defined by (1).

Lemma 1.1. Let $N$ be a numeraire and $Y$ a random variable such that $\mathrm{E}^{Q_{0}}\left[|Y| / S_{T}^{0}\right]<\infty$. Then

$$
N_{t} \mathrm{E}^{Q_{N}}\left[\begin{array}{c|c}
Y & \mathscr{T}_{t} \\
N_{T}
\end{array}\right]=S_{t}^{0} \mathrm{E}^{Q_{0}}\left[\begin{array}{c|c}
Y & \mathscr{T}_{t} \\
S_{T}^{0}
\end{array}\right]
$$

for all $t \in \mathscr{T}$.

Proof: We have

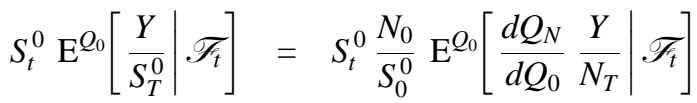

$$
\begin{aligned}
& =S_{t}^{0} \frac{N_{0}}{S_{0}^{0}} \mathrm{E}^{Q_{0}}\left[\begin{array}{c}
d Q_{N} \\
d Q_{0}
\end{array} \mid \mathscr{T}_{t}\right] \quad \mathrm{E}^{Q_{N}}\left[\begin{array}{c}
Y \\
N_{T}
\end{array} \mid \mathscr{F}_{t}\right]
\end{aligned}
$$

where the first equality follows from (1) and the second from a version of the Bayes rule. Now,

$$
S_{t}^{0} \begin{aligned}
& N_{0} \\
& S_{0}^{0}
\end{aligned} \mathrm{E}^{Q_{0}}\left[\begin{array}{c}
d Q_{N} \\
d Q_{0}
\end{array} \mid \mathscr{T}_{t}\right]=S_{t}^{0} \mathrm{E}^{Q_{0}}\left[\begin{array}{c}
N_{T} \\
S_{T}^{0}
\end{array} \mid \mathscr{T}_{t}\right]=N_{t}
$$

by (1) and the martingale property of $N / S^{0}$ under $Q_{0}$.

Applying this lemma to $Y=V_{T}^{\theta}$ with $\theta \in \Theta$, we see that $Q_{N} \in \mathbb{P}_{N}$. We call it the martingale measure obtained from $Q_{0}$ by change of numeraire. If $Q_{N}$ and $Q_{\tilde{N}}$ are obtained from $Q_{0}$ by changing the numeraire to $N$ and $\tilde{N}$, respectively, then (1) implies 


$$
\begin{aligned}
& d Q_{N} \\
& d Q_{\tilde{N}}
\end{aligned}=\begin{array}{ll}
N_{T} & \tilde{N}_{0} \\
N_{0} & \tilde{N}_{T}
\end{array} .
$$

Equations (1) and (2) are at the heart of the change-of-numeraire technique in derivative asset pricing; see Geman, El Karoui and Rochet (1995) for a detailed presentation of this technique, and Conze and Viswanathan (1991) for further results on the relationship between numeraires and martingale measures.

A contingent claim is a non-negative random variable $\Gamma$ on $(\Omega, \mathscr{F})$ such that $\Gamma / S_{T}^{0}$ is $Q_{0}$-integrable. A contingent claim is attainable if there exists a trading strategy $\theta \in \Theta$ that replicates the claim, i.e., that satisfies $V_{T}^{\theta}=\Gamma$. In this case, the portfolio value $V_{t}^{\theta}$ determines the time $t$ arbitrage price $\pi_{t}(\Gamma)$ of the claim. By the martingale property of normalised portfolio values, this price can be calculated as

$$
\pi_{t}(\Gamma)=S_{t}^{0} E^{Q_{0}}\left[\begin{array}{c|c}
\Gamma & \mathscr{F}_{t} \\
S_{T}^{0} &
\end{array}\right],
$$

that is, without reference to the replicating strategy. More generally, consider an arbitrary measure $Q \in \mathbb{P}_{0}$ under which $\Gamma / S_{T}^{0}$ is integrable. Independent of whether $\Gamma$ is attainable or not,

$$
\pi_{t}^{Q}(\Gamma)=S_{t}^{0} E^{Q}\left[\begin{array}{c|c}
\Gamma & \mathscr{T} \\
S_{T}^{0} &
\end{array}\right]
$$

is called the price under $Q$ of the claim at time $t$. Jacka (1992) shows that a contingent claim $\Gamma$ is attainable if and only if it has the same initial price $\pi_{0}^{Q}(\Gamma)$ under all $Q \in \mathbb{P}_{0}$ for which both $d Q_{0} / d Q$ and $d Q / d Q_{0}$ are bounded. Moreover, he shows that for bounded $\Gamma / S_{T}^{0}$, the attainability of the claim does not depend on which reference measure $Q_{0}$ was used to define the space of admissible trading strategies.

\section{European call options}

Consider an option to receive at time $T$ one unit of asset 1 in exchange for $K>0$ units of asset 0 . This is a slight generalisation of a classical European call option. Indeed, the latter is just the special case where asset 0 is a default-free zero-coupon bond of maturity $T$.

The option has the following value at the exercise date:

$$
\Gamma=\left[S_{T}^{1}-K S_{T}^{0}\right]^{+}=\left(S_{T}^{1}-K S_{T}^{0}\right) 1_{\mathscr{C}}
$$

where

$$
\mathscr{E}=\left\{\omega \in \Omega: S_{T}^{1}(\omega)>K S_{T}^{0}(\omega)\right\}
$$

is the event that the option ends 'in the money' and is exercised, and $1_{\mathscr{\delta}}$ is the corresponding indicator function.

It is well known that the price of a European option can be expressed in terms of exercise probabilities calculated under certain martingale measures. A variant of the following result was derived by El Karoui and Rochet (1989). We shall obtain this result as a special case of Proposition 2.2 below. 
Proposition 2.1. The option price under $Q_{0}$ is

$$
\pi_{t}^{Q_{0}}(\Gamma)=S_{t}^{1} Q_{1}\left(\mathscr{E} \mid \mathscr{F}_{t}\right)-K S_{t}^{0} Q_{0}\left(\mathscr{E} \mid \mathscr{F}_{t}\right)
$$

where $Q_{1} \in \mathbb{P}_{1}$ is the measure obtained from $Q_{0}$ by changing the numeraire to asset 1 .

We want to derive a similar decomposition of the option price for the case that the relative price $X=S^{1} / S^{0}$ is bounded.

Assumption (B) There are constants $0 \leq \ell<u \leq+\infty$ such that

$$
\ell S_{t}^{0}<S_{t}^{1}<u S_{t}^{0}
$$

for all $t \in \mathscr{T}$.

In view of this assumption, we shall consider the above option for $\ell<K<u$ only.

We introduce two portfolios, the first of which is long one unit of asset 0 and short $u^{-1}$ units of asset 1 , while the second is long one unit of asset 1 and short $\ell$ units of asset 0 (of course, $u^{-1}$ is understood to be zero if $u=+\infty$ ). Let

$$
U=S^{0}-u^{-1} S^{1}
$$

and

$$
L=S^{1}-\ell S^{0}
$$

denote the corresponding value processes. Under Assumption (B), these are positive processes, hence numeraires.

Proposition 2.2. Under Assumption (B), the option price under $Q_{0}$ is

$$
\pi_{t}^{Q_{0}}(\Gamma)=\frac{1}{1-u^{-1} \ell}\left\{\left(1-u^{-1} K\right) L_{t} Q_{L}\left(\mathscr{E} \mid \mathscr{T}_{t}\right)-(K-\ell) U_{t} Q_{U}\left(\mathscr{E} \mid \mathscr{F}_{t}\right)\right\}
$$

where $Q_{U} \in \mathbb{P}_{U}$ and $Q_{L} \in \mathbb{P}_{L}$ are the measures obtained from $Q_{0}$ by changing the numeraire to $U$ and $L$, respectively.

Proof: $\Gamma / S_{T}^{0}$ is clearly integrable under $Q_{0}$, so $\pi_{t}^{Q_{0}}(\Gamma)$ is well defined. Noting that

$$
S_{T}^{1}-K S_{T}^{0}=\frac{\left(1-u^{-1} K\right) L_{T}-(K-\ell) U_{T}}{1-u^{-1} \ell},
$$

we have

$$
\begin{aligned}
& \pi_{t}^{Q_{0}}(\Gamma)=\frac{1}{1-u^{-1} \ell}\left\{\left(1-u^{-1} K\right) S_{t}^{0} \mathrm{E}^{Q_{0}}\left[\begin{array}{cc|c}
L_{T} & 1_{\mathscr{O}} & \mathscr{T}_{t} \\
S_{T}^{0}
\end{array}\right]\right. \\
& \left.-(K-\ell) S_{t}^{0} \mathrm{E}^{Q_{0}}\left[\begin{array}{ll|l}
U_{T} & 1 \mathscr{E} & \mathscr{T}_{t} \\
S_{T}^{0} & &
\end{array}\right]\right\} .
\end{aligned}
$$

Lemma 1.1 now implies 


$$
S_{t}^{0} \mathrm{E}^{Q_{0}}\left[\begin{array}{c|c|c}
L_{T} & 1_{\mathscr{E}} & \mathscr{F}_{t} \\
S_{T}^{0}
\end{array}\right]=L_{t} \mathrm{E}^{Q_{L}}\left[1_{\mathscr{E}} \mid \mathscr{F}_{t}\right]
$$

and

$$
S_{t}^{0} \mathrm{E}^{Q_{0}}\left[\begin{array}{cc|c}
U_{T} & 1 \\
S_{T}^{0} & 1_{\mathscr{C}} & \mathscr{F}_{t}
\end{array}\right]=U_{t} \mathrm{E}^{Q_{U}}\left[1_{\mathscr{E}} \mid \mathscr{F}_{t}\right] .
$$

This is the desired result.

We have again expressed the call price as a function of certain exercise probabilities, this time evaluated under martingale measures associated with the numeraires $U$ and $L$. Setting $\ell=0$ and $u=+\infty$ yields Proposition 2.1.

\section{Models with a quadratic diffusion coefficient}

The following assumption postulates that after a change of measure, the process of relative asset prices is a diffusion with quadratic diffusion coefficient. Let constants $\sigma>0$ and $0 \leq \ell<u \leq+\infty$ be given.

Assumption (Q) There exists a $Q_{0}$-Wiener process $W^{0}$ such that the process of relative asset prices $X=S^{1} / S^{0}$ solves the stochastic differential equation

$$
d X_{t}=\sigma\left(X_{t}-\ell\right)\left(1-u^{-1} X_{t}\right) d W_{t}^{0}
$$

with initial value $\ell<X_{0}<u$.

Standard results from the theory of stochastic processes imply that the above stochastic differential equation has in fact a solution. This solution is unique both in the strong and weak sense, satisfies Assumption (B) and is a martingale; see for example Revuz and Yor (1991) and Karlin and Taylor (1981). In particular, $Q_{0}$ is indeed a martingale measure with respect to asset 0 .

Note that the lognormal dynamics of Black and Scholes (1973), Merton (1973), Margrabe (1978) and others are obtained as the special case where $\ell=0$ and $u=+\infty$.

\subsection{Characterisation}

Let $Q_{U} \in \mathbb{P}_{U}$ and $Q_{L} \in \mathbb{P}_{L}$ be the measures obtained from $Q_{0}$ by changing the numeraire to $U=S^{0}-u^{-1} S^{1}$ and $L=S^{1}-\ell S^{0}$, respectively, and define $\hat{\sigma}=\left(1-u^{-1} \ell\right) \sigma$.

Lemma 3.1. Assumption (Q) is equivalent to each of the following two properties:

(i) There exists a $Q_{U}$-Wiener process $W^{U}$ such that the process $Y=L / U$ solves $d Y_{t}=\hat{\sigma} Y_{t} d W_{t}^{U}$ with initial value $Y_{0}>0$.

(ii) There exists a $Q_{L}$-Wiener process $W^{L}$ such that the process $Z=U / L$ solves $d Z_{t}=\hat{\sigma} Z_{t} d W_{t}^{L}$ with initial value $Z_{0}>0$. 
Proof: Suppose Assumption (Q) holds. By Itô's lemma,

$$
d Y_{t}=\hat{\sigma} Y_{t}\left\{d W_{t}^{0}+\tilde{\sigma}\left(X_{t}-\ell\right) d t\right\}
$$

where $\tilde{\sigma}=u^{-1} \sigma$. Define a process $W^{U}$ by $d W_{t}^{U}=d W_{t}^{0}+\tilde{\sigma}\left(X_{t}-\ell\right) d t$ with $W_{0}^{U}=0$. We want to show that $W^{U}$ is a Wiener process under $Q_{U}$. By equation (2),

$$
\frac{d Q_{U}}{d Q_{0}}=\frac{U_{T}}{U_{0}} S_{0}^{0} S_{T}^{0}=\frac{1-u^{-1} X_{T}}{1-u^{-1} X_{0}} .
$$

On the other hand,

$$
\frac{d\left[1-u^{-1} X_{t}\right]}{1-u^{-1} X_{t}}=-\tilde{\sigma}\left(X_{t}-\ell\right) d W_{t}^{0},
$$

hence, by the formula for the martingale exponential,

$$
1-u^{-1} X_{t}=\left(1-u^{-1} X_{0}\right) \exp \left(-\tilde{\sigma} \int_{0}^{t}\left(X_{s}-\ell\right) d W_{s}^{0}-\frac{\tilde{\sigma}^{2}}{2} \int_{0}^{t}\left(X_{s}-\ell\right)^{2} d s\right) .
$$

In particular,

$$
\frac{d Q_{U}}{d Q_{0}}=\exp \left(-\tilde{\sigma} \int_{0}^{T}\left(X_{s}-\ell\right) d W_{s}^{0}-\frac{\tilde{\sigma}^{2}}{2} \int_{0}^{T}\left(X_{s}-\ell\right)^{2} d s\right) .
$$

The Girsanov theorem now implies that $W^{U}$ is indeed a $Q_{U}$-Wiener process; cf. Revuz and Yor (1991).

Next, we want to show that (i) implies (ii). Let $W^{U}$ be a $Q_{U}$-Wiener process as in the statement of the lemma. As $Z=1 / Y$, we have $d Z_{t}=\hat{\sigma} Z_{t}\left(-d W_{t}^{U}+\hat{\sigma} d t\right)$ by Itô's lemma. On the other hand,

$$
\frac{d Q_{L}}{d Q_{U}}=\frac{U_{0}}{L_{0}} \frac{L_{T}}{U_{T}}=\frac{Y_{T}}{Y_{0}}=\exp \left(\hat{\sigma} W_{T}^{U}-\frac{1}{2} \hat{\sigma}^{2} T\right),
$$

so the process $W^{L}$ defined by $d W_{t}^{L}=-d W_{t}^{U}+\hat{\sigma} d t$ with $W_{0}^{L}=0$ is a Wiener process under $Q_{L}$ according to the Girsanov theorem.

To prove the implication (ii) $\Rightarrow(\mathrm{Q})$, suppose we have $W^{L}$ as in the lemma. Itô's lemma yields

$$
d X_{t}=\sigma\left(X_{t}-\ell\right)\left(1-u^{-1} X_{t}\right)\left\{-d W_{t}^{L}+\sigma\left(1-u^{-1} X_{t}\right) d t\right\} .
$$

Let $W^{0}$ be the process defined by $d W_{t}^{0}=-d W_{t}^{L}+\sigma\left(1-u^{-1} X_{t}\right) d t$ with $W_{0}^{0}=0$. As

$$
\frac{d\left[X_{t}-\ell\right]}{X_{t}-\ell}=-\sigma\left(1-u^{-1} X_{t}\right) d W_{t}^{L}+\sigma^{2}\left(1-u^{-1} X_{t}\right)^{2} d t,
$$

the formula for the martingale exponential implies

$$
X_{t}-\ell=\left(X_{0}-\ell\right) \exp \left(-\sigma \int_{0}^{t}\left(1-u^{-1} X_{s}\right) d W_{s}^{L}+\frac{\sigma^{2}}{2} \int_{0}^{t}\left(1-u^{-1} X_{s}\right)^{2} d s\right)
$$

and 


$$
\frac{d Q_{0}}{d Q_{L}}=\frac{X_{0}-\ell}{X_{T}-\ell}=\exp \left(\sigma \int_{0}^{t}\left(1-u^{-1} X_{s}\right) d W_{s}^{L}-\frac{\sigma^{2}}{2} \int_{0}^{t}\left(1-u^{-1} X_{s}\right)^{2} d s\right) .
$$

By the Girsanov theorem, $W^{0}$ is a Wiener process under $Q_{0}$.

Thus, Assumption (Q) holds if and only if there is a change of measure that makes the process $Y$ (or $Z$ ) a driftless geometric Brownian motion whose 'volatility' (i.e., instantaneous standard deviation of returns) is $\hat{\sigma}$.

\subsection{The option price}

Let $\left(\mathscr{G}_{t}\right)_{t \in \mathscr{T}}$ be the augmention of the filtration generated by the process $X$, and set $\mathscr{G}=\mathscr{G}_{T}$. The following result is well known.

Proposition 3.1. Under Assumption (Q), any contingent claim $\Gamma$ with $\mathscr{G}$-measurable normalised payoff $\Gamma / S_{T}^{0}$ is attainable.

Proof: The proposition is an immediate consequence of the martingale representation property of $X$ on $\left(\Omega, \mathscr{G}, Q_{0},\left(\mathscr{G}_{t}\right)_{t \in \mathscr{T}}\right)$; see Revuz and Yor (1991).

This guarantees in particular attainability of the option to receive one unit of asset 1 in exchange for $K$ units of asset 0 , as its normalised payoff $\left[S_{T}^{1}-K S_{T}^{0}\right]^{+} / S_{T}^{0}=\left[X_{T}-K\right]^{+}$is clearly measurable with respect to $\mathscr{G}$. For $u<+\infty$, moreover, the normalised payoff of the option is bounded, so attainability does not depend on which reference measure was chosen to define the space of admissible trading strategies; cf. Jacka (1992). To show this in the case $u=+\infty$, we can consider a generalised put option (an option to give up one unit of asset 1 in exchange for $K$ units of asset 0 ) whose normalised payoff $\left[K-X_{T}\right]^{+}$ is always bounded, and then use put-call parity.

Our calculation of the option price is based on Lemma 3.1 and the fact that the exercise event $\mathscr{E}=\left\{\omega \in \Omega: X_{T}(\omega)>K\right\}$ can be characterised fully in terms of the random variables $Y_{T}=L_{T} / U_{T}$ and $Z_{T}=U_{T} / L_{T}$. In fact, $Y_{T}=$ $\left(X_{T}-\ell\right) /\left(1-u^{-1} X_{T}\right)$ is a monotonically increasing function of $X_{T}$, and $Z_{T}=$ $\left(1-u^{-1} X_{T}\right) /\left(X_{T}-\ell\right)$ is monotonically decreasing in $X_{T}$, hence

$$
\mathscr{E}=\left\{\omega \in \Omega: Y_{T}(\omega)>\begin{array}{c}
K-\ell \\
1-u^{-1} K
\end{array}\right\}=\left\{\omega \in \Omega: Z_{T}(\omega)<\frac{1-u^{-1} K}{K-\ell}\right\} .
$$

Let $\Phi$ denote the standard normal distribution function.

Proposition 3.2. Under Assumption (Q), the option to receive one unit of asset 1 in exchange for $K$ units of asset 0 is attainable. For $\ell<K<u$, its time $t$ arbitrage price is

$\pi_{t}(\Gamma)=\frac{1}{1-u^{-1} \ell}\left\{\left(1-u^{-1} K\right)\left(S_{t}^{1}-\ell S_{t}^{0}\right) \Phi\left(e_{t}^{+}\right)-(K-\ell)\left(S_{t}^{0}-u^{-1} S_{t}^{1}\right) \Phi\left(e_{t}^{-}\right)\right\}$

where 


$$
e_{t}^{ \pm}=\frac{1}{\hat{\sigma} \sqrt{ } T-t}\left[\log \frac{S_{t}^{1}-\ell S_{t}^{0}}{S_{t}^{0}-u^{-1} S_{t}^{1}}-\log \frac{K-\ell}{1-u^{-1} K} \pm \frac{1}{2} \hat{\sigma}^{2}(T-t)\right]
$$

and $\hat{\sigma}=\left(1-u^{-1} \ell\right) \sigma$.

Proof: We want to apply Proposition 2.2, so let $Q_{U}$ and $Q_{L}$ be the measures obtained from $Q_{0}$ by changing the numeraire to $U$ and $L$, respectively. To calculate the probability of exercise under $Q_{U}$ and $Q_{L}$, let $W^{U}$ and $W^{L}$ be Wiener processes as in Lemma 3.1, so that

$$
Y_{t}=Y_{0} \exp \left(\hat{\sigma} W_{t}^{U}-\frac{1}{2} \hat{\sigma}^{2} t\right)
$$

and

$$
Z_{t}=Z_{0} \exp \left(\hat{\sigma} W_{t}^{L}-\frac{1}{2} \hat{\sigma}^{2} t\right)
$$

by the formula for the martingale exponential.

The properties of the Wiener process $W^{U}$ now imply

$$
\begin{aligned}
Q_{U}\left(\mathscr{E} \mid \mathscr{F}_{t}\right) & =Q_{U}\left(Y_{T}>\frac{K-\ell}{1-u^{-1} K} \mid Y_{t}\right) \\
& =Q_{U}\left(\log Y_{T}-\log Y_{t}>\log \frac{K-\ell}{1-u^{-1} K}-\log Y_{t}\right) \\
& =Q_{U}\left(\hat{\sigma}\left(W_{T}^{U}-W_{t}^{U}\right)>\log \frac{K-\ell}{1-u^{-1} K}-\log Y_{t}+\frac{1}{2} \hat{\sigma}^{2}(T-t)\right) \\
& =\Phi\left(\frac{1}{\hat{\sigma} \sqrt{ } T-t}\left[\log Y_{t}-\log \frac{K-\ell}{1-u^{-1} K}-\frac{1}{2} \hat{\sigma}^{2}(T-t)\right]\right) .
\end{aligned}
$$

In the same way, we find

$$
\begin{aligned}
Q_{L}\left(\mathscr{E} \mid \mathscr{T}_{t}\right) & =Q_{L}\left(Z_{T}<\frac{1-u^{-1} K}{K-\ell} \mid Z_{t}\right) \\
& =Q_{L}\left(\hat{\sigma}\left(W_{T}^{L}-W_{t}^{L}\right)<\log \frac{1-u^{-1} K}{K-\ell}-\log Z_{t}+\frac{1}{2} \hat{\sigma}^{2}(T-t)\right) \\
& =\Phi\left(\frac{1}{\hat{\sigma} \sqrt{ } T-t}\left[\log Y_{t}-\log \frac{K-\ell}{1-u^{-1} K}+\frac{1}{2} \hat{\sigma}^{2}(T-t)\right]\right) .
\end{aligned}
$$

This completes the proof.

Standard arguments from Harrison and Pliska (1981) show that the trading strategy

$$
\begin{aligned}
& \theta_{t}^{0}=\frac{1}{1-u^{-1} \ell}\left\{-\left(1-u^{-1} K\right) \ell \Phi\left(e_{t}^{+}\right)-(K-\ell) \Phi\left(e_{t}^{-}\right)\right\} \\
& \theta_{t}^{1}=\frac{1}{1-u^{-1} \ell}\left\{\left(1-u^{-1} K\right) \Phi\left(e_{t}^{+}\right)+(K-\ell) u^{-1} \Phi\left(e_{t}^{-}\right)\right\}
\end{aligned}
$$

is admissible and replicates the option. 
For $\ell=0$ and $u=+\infty$, we obtain of course an option price formula as in Black and Scholes (1973), Merton (1973) and Margrabe (1978) with $\hat{\sigma}=\sigma$. Setting $u=+\infty$ but $\ell>0$ leads to a formula derived by Rubinstein (1983).

The result is easily extended to allow a time-dependent, but deterministic, parameter function $\sigma(t)>0$ in Assumption (Q). Lemma 3.1 then holds with $\hat{\sigma}$ replaced by $\hat{\sigma}(t)=\left(1-u^{-1} \ell\right) \sigma(t)$, and the term $\hat{\sigma} \sqrt{ } T-t$ in Proposition 3.2 must be replaced with

$$
\left(1-u^{-1} \ell\right) \sqrt{\int_{t}^{T} \sigma^{2}(s) d s .}
$$

The price of a generalised put option, that is, an option to give up one unit of asset 1 in exchange for $K$ units of asset 0 , can be calculated in the same way. Alternatively, one can use put-call parity.

\section{Options on bonds and interest rates}

This section shows how our earlier results apply to the pricing of options on discount bonds and so-called interest rate caplets, defined below.

Fix dates $T^{\prime}>T>0$ and let assets 0 and 1 be pure discount bonds without default risk, maturing at $T$ and $T^{\prime}$, respectively. Without loss of generality, their face values can be normalised to 1 , i.e., $S_{T}^{0}=1$ and $S_{T^{\prime}}^{1}=1$. Note that $X_{t}=S_{t}^{1} / S_{t}^{0}$ is the time $t$ forward price of bond 1 for delivery at time $T$.

In an economy with positive interest rates, the prices of these two bonds must satisfy

$$
\begin{array}{ll}
S_{t}^{0}<1 & \text { for } t<T, \\
S_{t}{ }^{1}<1 & \text { for } t<T^{\prime}
\end{array}
$$

and

$$
S_{t}^{1}<S_{t}^{0} \quad \text { for } t \leq T .
$$

In fact, (3) and (4) mean that the interest rate for a loan from $t$ to $T$ or $T^{\prime}$ is always positive, while (5) states that the forward interest rate, as seen at time $t$, for the period from $T$ to $T^{\prime}$ is positive. In particular, Assumption (B) must hold with $u=1$ and $\ell=0$. Note that for these values of the upper and lower bound, we have the numeraires $U=S^{0}-S^{1}$ and $L=S^{1}$, and the process $Z$ introduced earlier becomes

$$
Z=\frac{U}{L}=\frac{S^{0}-S^{1}}{S^{1}}=\frac{1}{X}-1 .
$$

Thus, $Z_{t}$ is the once compounded forward rate, as seen at time $t$, for a loan starting at $T$ and ending at $T^{\prime}$.

Consider a standard European call option written on bond 1 with exercise price $0<K<1$ and exercise date $T$. The call payoff at time $T$ is $\Gamma=\left[S_{T}^{1}-K\right]^{+}$. As $S_{T}^{0}=1$, this call can be considered as an option to receive one unit of bond 1 in exchange for $K$ units of bond 0 , so our earlier results apply. 
As a second contingent claim, we introduce a caplet that protects its holder against a rise of $Z_{T}$, the once compounded interest rate for the period from $T$ to $T^{\prime}$, above a given level $k>0$. Suppose that the face value of the caplet is 1 and that its payoff $\left[Z_{T}-k\right]^{+}$, which is known at time $T$, is paid out in arrears at time $T^{\prime}$. Then the time $T$ value of the caplet is $\gamma=S_{T}^{1}\left[Z_{T}-k\right]^{+}$.

Let a constant $\sigma>0$ be given.

Proposition 4.1. The following two properties are equivalent:

(a) There exist a measure $Q_{0}$ equivalent to $P$ and a $Q_{0}$-Wiener process $W^{0}$ such that the forward bond price process $X=S^{1} / S^{0}$ solves $d X_{t}=\sigma X_{t}\left(1-X_{t}\right) d W_{t}^{0}$ with initial value $0<X_{0}<1$.

(b) There exist a measure $Q_{1}$ equivalent to $P$ and a $Q_{1}$-Wiener process $W^{1}$ such that the forward rate process $Z=S^{0} / S^{1}-1$ solves $d Z_{t}=\sigma Z_{t} d W_{t}^{1}$ with initial value $Z_{0}>0$.

If (a) and (b) hold, then the bond option $\Gamma=\left[S_{T}^{1}-K\right]^{+}$with exercise price $0<K<1$ and the caplet $\gamma=S_{T}^{1}\left[Z_{T}-k\right]^{+}$with $k>0$ have the following time $t$ arbitrage prices:

$$
\pi_{t}(\Gamma)=(1-K) S_{t}^{1} \Phi\left(e_{t}^{+}\right)-K\left(S_{t}^{0}-S_{t}^{1}\right) \Phi\left(e_{t}^{-}\right)
$$

with

$$
e_{t}^{ \pm}=\frac{1}{\sigma \sqrt{ } T-t}\left[\log \frac{S_{t}^{1}}{S_{t}^{0}-S_{t}^{1}}-\log \frac{K}{1-K} \pm \frac{1}{2} \sigma^{2}(T-t)\right]
$$

and

$$
\pi_{t}(\gamma)=S_{t}^{1}\left\{Z_{t} \Phi\left(d_{t}^{+}\right)-k \Phi\left(d_{t}^{-}\right)\right\}
$$

with

$$
d_{t}^{ \pm}=\frac{1}{\sigma \sqrt{ } T-t}\left[\log \frac{Z_{t}}{k} \pm \frac{1}{2} \sigma^{2}(T-t)\right] \text {. }
$$

Proof: The equivalence (a) $\Leftrightarrow$ (b) is just the statement (Q) $\Leftrightarrow$ (ii) in Lemma 3.1 specialised to $\ell=0$ and $u=1$. Now suppose that (a) and (b) hold. The option price formula (6)-(7) then follows from Proposition 3.2. By Proposition 3.1, the caplet is attainable, and by the change-of-numeraire formula of Lemma 1.1, its arbitrage price can be calculated as

$$
\pi_{t}(\gamma)=S_{t}^{0} \mathrm{E}^{Q_{0}}\left[\begin{array}{c}
\gamma \\
S_{T}^{0}
\end{array} \mid \mathscr{F}_{t}\right]=S_{t}^{1} \mathrm{E}^{Q_{1}}\left[\begin{array}{c}
\gamma \\
S_{T}^{1}
\end{array} \mid \mathscr{F}_{t}\right]=S_{t}^{1} \mathrm{E}^{Q_{1}}\left[\left[Z_{T}-k\right]^{+} \mid \mathscr{F}_{t}\right] .
$$

As $Z$ is a driftless geometric Brownian motion under $Q_{1}$, the caplet price (8)-(9) now follows by a standard calculation.

Again, this result generalises in the usual way to time-dependent parameter functions $\sigma(t)>0$; see the remark after the proof of Proposition 3.2.

Bühler and Käsler (1989) were the first to propose a bond price model satisfying (a) and to derive the bond option formula (6)-(7). Caplet price formulae similar to (8)-(9), by contrast, have long been used by practitioners. In fact, it has 
been market practice to value caplets under the assumption that the underlying forward rate follows a lognormal process, leading to pricing formulae resembling the Black (1976) formula for futures options.

Sandmann et al. (1994) were the first to derive both bond option and caplet prices of the above type in a unified framework. They construct a term structure model where each of a finite number of annually compounded forward interest rates follows a lognormal diffusion process and satisfies a variant of property (b). Subsequently, their results have been extended by Brace et al. (1995) and Miltersen et al. (1997).

Bühler and Käsler (1989) and Sandmann et al. (1994) calculate bond option prices by solving the fundamental valuation PDE. ${ }^{5}$ Our probabilistic proof of the bond option formula (6)-(7) is new. ${ }^{6}$

Independently of the work reported here, Goldys (1995) has given an alternative probabilistic proof of the above bond option formula. In our notation, his main result can be rendered as follows. Suppose property (a) holds with a time dependent parameter function $\sigma(t)$, and let $g: \mathbb{R} \rightarrow \mathbb{R}_{+}$be a measurable function. Then

$$
\begin{aligned}
\mathrm{E}^{Q_{0}\left[g\left(X_{T}\right) \mid X_{t}\right]=} & e^{-\Sigma^{2}(t, T) / 8} \int_{-\infty}^{\infty} g\left(\left[1+\frac{1-X_{t}}{X_{t}} e^{-\xi}\right]^{-1}\right) \\
& \times\left(X_{t} e^{\xi / 2}+\left(1-X_{t}\right) e^{-\xi / 2}\right) \phi(\xi) d \xi
\end{aligned}
$$

where $\phi$ is the normal density function for mean 0 and variance $\Sigma^{2}(t, T)=$ $\int_{t}^{T} \sigma^{2}(s) d s$. Evaluating the integral for the function $g(x)=[x-K]^{+}$leads to the call price formula (6)-(7). Goldys' approach is more general than ours in that it allows arbitrary payoff profiles (besides, his method readily generalises to arbitrary bounds $\ell$ and $u$ ). Our approach, however, has the advantage of yielding a particularly simple and more transparent calculation in the case of standard options.

\section{Conclusion}

We have studied the pricing of a European type option to exchange one asset for another in the presence of strict upper and lower bounds on the relative price of these assets. Our first result shows how to decompose the option price in terms of two particular numeraire portfolios and the probabilities of exercise under the martingale measures associated with these numeraires. The second result is a characterisation of models where the relative asset price has a quadratic diffusion coefficient. Combining these results, we obtain a new, purely probabilistic

\footnotetext{
5 This derivation of the option price appeared first in Käsler (1991), a Ph.D. thesis written in German. It can also be found in Rady and Sandmann (1994).

${ }^{6}$ Our proof of the implication (b) $\Rightarrow(8)-(9)$, on the other hand, follows the probabilistic derivation of the caplet formula in Brace et al. (1995) and Miltersen et al. (1997).
} 
derivation of the option price for this type of underlying price dynamics. Moreover, these results unify the pricing of standard options on bonds and interest rates in certain lognormal term structure models.

\section{References}

Black, F.: The Pricing of Commodity Contracts. Journal of Financial Economics 3, 167-179 (1976)

Black, F., M. Scholes: The Pricing of Options and Corporate Liabilities. Journal of Political Economy 81, 637-654 (1973)

Brace, A., Gạtarek, D., Musiela, M.: The Market Model of Interest Rate Dynamics. Mathematical Finance 7, 127-154 (1997)

Bühler, W., Käsler, J.: Konsistente Anleihepreise und Optionen auf Anleihen. Working paper, Universität Dortmund, Germany (1989)

Conze, A., Viswanathan, R.: Probability Measures and Numeraires. Working paper, Université Paris Dauphine and Université Paris 6 (1991)

El Karoui, N., Rochet, J.-C.: A Pricing Formula for Options on Coupon Bonds. Working Paper, GREMAQ, Université Toulouse 1, France (1989)

Geman, H.: L'Importance de la Probabilité "Forward Neutre" dans une Approche Stochastique des Taux d'Intérêt. Working paper, ESSEC, France (1989)

Geman, H., El Karoui, N., Rochet, J.-C.: Changes of Numéraire, Changes of Probability Measure and Option Pricing. Journal of Applied Probability 32, 443-458 (1995)

Goldys, B.: On Pricing Interest Rate Derivatives when the Forward Libor Rates are Lognormal. Working paper, School of Mathematics, University of New South Wales, Australia (1995)

Harrison, J.M., Pliska, S.R.: Martingales and Stochastic Integrals in the Theory of Continuous Trading. Stochastic Processes and their Applications 11, 215-260 (1981)

Ingersoll, J.E.: Contingent Foreign Exchange Contracts. Working paper, Yale School of Management (1989a)

Ingersoll, J.E.: Valuing Foreign Exchange Derivatives with a Bounded Exchange Rate Process. Working paper, Yale School of Management (1989b)

Jacka, S.D.: A Martingale Representation Result and an Application to Incomplete Financial Markets. Mathematical Finance 2(4), 239-250 (1992)

Jamshidian, F.: Pricing of Contingent Claims in the One-Factor Term Structure Model. Working paper, Merrill Lynch Capital Markets, New York (1987)

Käsler, J.: Optionen auf Anleihen. Ph.D. Thesis, Universität Dortmund, Germany (1991)

Karlin, S., Taylor, H.M.: A Second Course in Stochastic Processes. New York: Academic Press 1981

Margrabe, W.: The Value of an Option to Exchange One Asset for Another. Journal of Finance 33, 177-198 (1978)

Merton, R.C.: Theory of Rational Option Pricing. Bell Journal of Economics and Management 4, 141-183 (1973)

Miltersen, K.R., Sandmann, K., Sondermann, D.: Closed Form Solutions for Term Structure Derivatives with Log-Normal Interest Rates. Journal of Finance 52(1), 409-430 (1997)

Rady, S., Sandmann, K.: The Direct Approach to Debt Option Pricing. Review of Futures Markets 13(2), 461-514 (1994)

Revuz, D., Yor, M.: Continuous Martingales and Brownian Motion. New York: Springer 1991

Rubinstein, M.: Displaced Diffusion Option Pricing. Journal of Finance 38(1), 213-217 (1983)

Sandmann, K., Sondermann, D., Miltersen, K.R.: Closed Form Term Structure Derivatives in a HeathJarrow-Morton Model with Log-Normal Annually Compounded Interest Rates. Proceedings of the Seventh Annual European CBOT Futures Research Symposium, Bonn (Germany), September 1994, pp 145-164

Sondermann, D.: Currency Options: Hedging and Social Value. European Economic Review 31, 246-256 (1987)

Sondermann, D.: Option Pricing with Bounds on the Underlying Security. In: Rudolph, B., Wilhelm, J. (eds.): Bankpolitik, finanzielle Unternehmensführung und die Theorie der Finanzmärkte. Berlin: Duncker \& Humblot 1988, pp 421-442 